\title{
La vie d'artiste ou le défi de la représentation
}

An artist's life or the challenge of the performance

\section{Laurent Aubert}

\section{OpenEdition}

\section{Journals}

Édition électronique

URL : http://journals.openedition.org/ethnomusicologie/1061

ISSN : 2235-7688

Éditeur

ADEM - Ateliers d'ethnomusicologie

Édition imprimée

Date de publication : 1 décembre 1996

Pagination : 23-52

ISBN : 978-2-8257-0559-9

ISSN : $1662-372 \mathrm{X}$

Référence électronique

Laurent Aubert, «La vie d'artiste ou le défı de la représentation », Cahiers d'ethnomusicologie [En ligne] 9 | 1996, mis en ligne le 05 janvier 2012, consulté le 19 avril 2019. URL : http:// journals.openedition.org/ethnomusicologie/1061

Ce document a été généré automatiquement le 19 avril 2019

Tous droits réservés 


\title{
La vie d'artiste ou le défi de la représentation
}

\author{
An artist's life or the challenge of the performance
}

\author{
Laurent Aubert
}

1 Paris, 21 septembre 1994. Nous sommes dans un studio d'enregistrement de Montmartre, attendant l'arrivée d'un groupe de musiciens maliens. Tous griots et émigrés en région parisienne, ils appartiennent à des familles très respectées dans leur pays, tant pour leur fidélité à l'héritage historico-musical de leurs ancêtres qu'en raison de leur connaissance du répertoire traditionnel et de leur talent d'interprètes. Leur prestige dans la diaspora est manifeste et, suite à quelques tournées de concerts en France et dans les pays voisins, ils commencent à jouir d'une certaine réputation parmi les amateurs européens de musiques africaines. Le projet est donc de réaliser avec eux un disque, pour lequel un de leurs amis français nous suggère le titre « Musique classique bambara du Mali », ceci afin de souligner l'estime due à la haute culture incarnée par ces artistes ${ }^{1}$.

2 Les musiciens arrivent maintenant dans le studio et commencent à s'installer, à déballer et à accorder leurs instruments; parmi ceux-ci nous découvrons la présence d'une superbe guitare électrique extra-plate rouge vif. Surpris, nous leur demandons si sa présence est indispensable, étant donné la nature du disque que nous envisageons de produire avec eux. «Ah! C'était pas prévu? s'étonne le guitariste. Pas de problème, on peut prendre celle-là, elle va aussi très bien ", nous dit-il en sortant une guitare à douze cordes de sa housse. « Mon père en jouait déjà avant moi ; c'est une guitare traditionnelle africaine ». L'un de nous lui demande s'il ne jouera pas aussi du petit luth ngoni, si fréquent chez les griots bambara. «Bien sûr, j'en ai même deux ici, répond-il fièrement, un normal et un spécial! » Le «normal » est effectivement un ngoni ordinaire à quatre cordes, mais muni d'une prise de micro jack dans sa caisse de résonance, et le « spécial » un instrument de son invention à sept cordes, sur lequel il a développé une technique de jeu lui permettant d'exécuter seul deux parties de ngoni « normaux ». 
3 Après une substantielle palabre, nous convenons que la guitare « folk » et les deux ngoni seront utilisés, mais pas la guitare électrique car sa présence voilerait les timbres de la kora, du balafon et de la flûte bambara. Cette solution semble convenir à tout le monde, et l'enregistrement se déroule ensuite dans les meilleures conditions.

4 Il ressort de cette anecdote que le choix d'un instrument ou d'un autre n'est pas nécessairement significatif pour un musicien, et notamment pour un musicien traditionnel comme un griot d'Afrique occidentale. Habitué à répondre à la demande, il semble disposé à se plier aux requêtes de ces nouveaux patrons que sont les responsables artistiques européens. Ce n'est pas tant son attitude face à la musique qui change avec le contexte que la nature des sollicitations dont il est l'objet, que celles-ci le poussent à une expression conservatrice ou, au contraire, à une démarche résolument moderne, voire transculturelle. Il n'en va en revanche pas toujours de même pour nous, spécialistes, promoteurs ou amateurs occidentaux de musiques traditionnelles, qui en avons souvent une image préconçue, forgée par l'idée que nous nous faisons de ce que devraient être ces musiques et leurs interprètes.

5 Le terme de «musiques traditionnelles » est aujourd'hui entré dans le langage courant pour définir un domaine comportant ses circuits de production, son public et ses exigences propres. Avant d'aborder la question de la représentation de ces musiques, et notamment le phénomène $\mathrm{du}$ concert, qui, depuis quelques années, connaît un développement significatif, il peut être utile de tenter une évaluation globale du concept de musiques traditionnelles, afin de déterminer, d'une part, s'il recouvre toujours une catégorie homogène et identifiable en tant que telle et, d'autre part, quelles sont, le cas échéant, les frontières qui la séparent d'autres catégories musicales.

De nombreuses personnes s'accordent sur l'idée que les musiques traditionnelles seraient des manifestations identitaires, distinctes en tant que telles des « produits dérivés » que sont par exemple les musiques dites folkloriques ou celles participant aux courants actuels de la world music. Une musique folklorique se démarquerait ainsi de ses sources traditionnelles par son aspect «arrangé », «commercialisé », "officiel », voire «nationaliste » résultant de l'intervention d'agents externes, alors que le label de world music s'appliquerait essentiellement aux expériences interculturelles de "fusion » dans le domaine de la musique populaire, expériences suscitées par la rencontre de musiciens d'origines diverses et par l'intégration d'instruments et de sonorités «exotiques » à l'appareillage électronique de la production musicale occidentale actuelle ( $c f$. Aubert 1991c). Si l'on voulait résumer l'opinion courante sur ces trois catégories, on pourrait dire que les musiques traditionnelles sont perçues comme étant authentiques, les musiques folkloriques éclectiques, et la world music syncrétique.

\section{La tradition en question : un problème de limites}

7 « Tradition ne veut pas dire simplement fixation stylistique et ne dépend pas seulement d'un consensus général », écrivait Ananda Coomaraswamy dans un article sur la nature de l'art traditionnel, qu'il opposait en tant que tel à l'art « académique » ou à celui « qui est à la mode ». "L'art traditionnel est ordonné à une fin particulière et emploie des moyens déterminés pour parvenir à sa fin; il est transmis de maitre à élève depuis un passé immémorial » (1990: 142). Selon cet auteur, un art traditionnel comporte donc un style propre, caractérisé par une série de critères relatifs à son esthétique; il fait l'objet d'un consensus partagé par la société ou le groupe au sein duquel il se manifeste; il a une fin, 
une raison d'être dans ce cadre, et il y est considéré comme efficace, c'est-à-dire doté non seulement d'une fonction, mais de pouvoirs, à un niveau ou un autre ; cette efficacité se manifeste, y compris sur les plans psychologique et symbolique, par l'application d'une série de moyens, de règles et de techniques spécifiques; la conservation de l'ensemble de ces données est garantie notamment par des modes de transmission adéquats; les arts traditionnels sont enfin caractérisés par l'ancienneté des principes qu'ils mettent en œuvre, sinon nécessairement de toutes les modalités de leurs expressions.

Un ouvrage tel que celui récemment publié par Jean During sur le sens de la tradition dans l'Orient musical étudie la question de façon approfondie. L'auteur y examine notamment " comment la tradition se détermine en s'opposant à d'autres formes, en les écartant, bref en exerçant son pouvoir, et donc en s'appuyant sur un pouvoir» (1994: 25). Dans l'intention de déterminer les composantes communes aux musiques traditionnelles, relatives tant à leur objet (la musique) qu'à leur sujet (l'homme, ses structures mentales et comportementales, son idéologie, son éthique, etc.), il dégage une série d'indices et de niveaux de pertinence partagés par ces musiques: 1 . la tradition comme processus de transmission ; 2 . le contenu et les formes ; 3 . les moyens de production ; 4 . les conditions de performance ${ }^{2}$ et d'audition; 5 . le contexte social et culturel ; 6 . le sens et les valeurs manifestés par la musique (cf. ibid. : 30-32).

Il n'est pas inutile de rappeler ici quelques résultats, déjà formulés ailleurs, d'une enquête menée auprès d'un échantillon représentatif de musiciens d'origines diverses, mais se considérant tous comme rattachés à une tradition. La synthèse de ces données fait ressortir de multiples axes de convergence qui, dans leur ensemble, viennent confirmer l'analyse de During. Les commentaires de la plupart de ces musiciens sur leur propre tradition musicale nous permettent de dégager un certain nombre de traits partagés par l'ensemble de ces musiques :

"- elles sont d'origine ancienne et fidèles à leurs sources dans leurs principes, sinon toujours nécessairement dans leurs formes et leurs occasions de jeu ;

- elles sont basées sur une transmission orale de leurs règles, de leurs techniques et de leurs répertoires ;

- elles sont liées à un contexte culturel, dans le cadre duquel elles ont une place et, la plupart du temps, une fonction précises ;

- elles sont porteuses d'un ensemble de valeurs et de vertus qui leur confèrent leur sens et leur efficacité au sein de ce contexte ;

- elles sont enfin liées à un réseau de croyances et de pratiques, parfois rituelles, dont elles tirent leur substance et leur raison d'être »(Aubert $1995: 18)^{3}$.

Pour fondée qu'elle soit, une évaluation globale du champ des musiques traditionnelles s'expose néanmoins à la question de leurs limites ${ }^{4}$. La démarcation entre les formes musicales relevant manifestement d'une tradition et celles qui s'en écartent apparaît plutôt, en particulier dans le contexte contemporain, comme une sorte de no man's land difficile à définir du fait de la perméabilité croissante des frontières culturelles et de l'interpénétration des genres qui en résulte. La distinction, au sein d'une musique donnée, entre ses ingrédients endogènes et ceux d'origine externe n'est en outre pas toujours aisément discernable, ni même pertinente ; toute culture est en effet susceptible d'absorber les apports extérieurs dans la mesure de leur compatibilité, mais aussi de s'intégrer à un cercle plus large et englobant ou de se dissoudre progressivement au contact d'influences corrosives. La question qu'on peut se poser à cet égard est double : jusqu'à quel stade de modernisation une musique peut-elle encore être considérée comme traditionnelle ? Et, corollairement, à partir de quel degré d'ancienneté peut-on estimer qu'une musique devient traditionnelle? Le blues est-il par exemple encore une forme 
traditionnelle parce qu'on y retrouve des réminiscences permettant de le rattacher à la tradition des griots et autres ménestrels africains, ou l'est-il déjà du fait que son existence, sous une forme relativement homogène, est déjà vieille de plus d'un siècle ? Y a-t-il en outre une catégorie de blues demeurée traditionnelle - celui d'une Bessie Smith ou d'un Big Bill Broonzy, par exemple - et d'autres qui cesseraient de l'être, du fait de l'introduction d'instruments électriques, de la commercialisation du genre ou de son appropriation par des Blancs? B.B. King et Chuck Berry sont-ils ainsi des bluesmen traditionnels? Et Elvis Presley, Eric Clapton ou Johnny Hallyday lorsqu'ils s'adonnent à cette musique ? Ils le pensent en tout cas tous !...

De nombreuses personnes estiment que la notion de tradition s'oppose en tant que telle à toute espèce de développement ou d'évolution. Pour elles, une forme traditionnelle est ainsi par définition une forme conservatrice, figée, incapable d'évoluer, voire rétrograde et réactionnaire. Cette opinion est néanmoins contredite par les faits et, lorsqu'elle s'applique aux manifestations de sociétés différentes de la nôtre, elle témoigne d'une sorte d'ethnocentrisme culturel qui est elle-même de nature réactionnaire, et que l'historien Tzvetan Todorov dénonce en ces termes: «Nous avons l'impression qu'une culture se développe, croyant porter par là un jugement objectif la concernant ; en réalité, tout ce dont nous témoignons est qu'elle se meut dans la même direction que nous. Ou bien, au contraire, nous croyons qu'une autre stagne : là encore, illusion d'optique, nous ne désignons en fait que la différence de direction entre notre mouvement et le sien » (1989: 98). Une musique traditionnelle n'est ainsi en aucun cas l'image d'une quelconque pureté originelle, ni celle d'un passé musical demeuré intact ; vivante et donc soumise aux changements comme n'importe quel organisme, elle exprime au contraire toujours son époque, manifestant les confluences et les étapes ayant marqué ses productions.

11 Le plus grand enseignement des musiques traditionnelles, telles que nous pouvons les apprécier aujourd'hui dans leur immense diversité, est peut-être qu'elles nous rappellent, par la vision du monde et de l'homme dont chacune procède, que toute musique, en tant que "son humainement organisé ${ }^{5}$, exprime en mode synthétique les valeurs d'une société ou d'un groupe humain. Je dirais même qu'elle les exprime nécessairement, faute de quoi une société ne serait pas ce qu'elle est et une musique ne serait pas de la musique. La meilleure définition de la tradition en musique est peut-être en définitive celle qui m’a un jour été donnée par un Gitan andalou, guitariste flamenco : «La tradition », me disaitil, « c'est une mère qui chante une berceuse à son bébé ; tout est là ! »

\section{Le paradoxe du concert ou l'évocation de la tradition}

12 Depuis quelques années, le goût pour les musiques traditionnelles du monde s'est développé en Occident avec une ampleur spectaculaire. Les théâtres qui les programment font régulièrement salle comble; chaque année, de nouveaux réseaux et de nouveaux festivals dédiés à ce vaste domaine surgissent un peu partout. La popularité acquise par certains genres musicaux offre à leurs interprètes les plus doués des occasions de s'intégrer à ces circuits et de jouir d'une notoriété appréciable ; elle permet en outre, par effet de proximité, d'élargir progressivement le champ d'appréciation du public à des genres voisins et à des expressions demeurées jusqu'à récemment à peu près inconnues.

Dans son ensemble, ce courant culturel est caractérisé par son exigence d'authenticité . Ce n'est pas a priori le potentiel spectaculaire d'une forme artistique qui attire les amateurs des musiques traditionnelles, ni l'usage qui peut en être fait dans une 
perspective de fusion interculturelle, mais avant tout la musique en soi, pour sa valeur intrinsèque et en tant que fait culturel signifiant. Ils s'attendent à ce qu'une musique manifestant une tradition soit représentée d'une façon inaltérée ou, du moins, analogue à sa réalité originelle, notamment en ce qui concerne ses formes et ses structures de jeu et leurs exigences spatio-temporelles. Lorsqu'elle est inévitable, la mise en scène s'y limitera en principe au minimum nécessaire à la compréhension du répertoire présenté et à la valorisation de ses différents niveaux sémantiques ; éventuellement aussi à la suggestion de son milieu d'origine, autant que possible au moyen des artifices et des matériaux qui lui sont propres.

Dès lors qu'elle est exportée, une musique normalement ancrée dans un type de contexte et de situation spécifique est confrontée à la question de son sens hors de ses structures de référence. Son transfert implique un décalage qui, selon les points de vue, peut être considéré en soi comme une sorte de trahison, ou en tout cas comme une distorsion par rapport à ses circonstances ordinaires de jeu. Aussi réel soit-il, ce risque ne se manifeste cependant pas dans tous les cas avec la même sévérité; il convient à cet effet de distinguer différentes catégories musicales, chorégraphiques et théâtrales, dont le déplacement hors contexte et la représentation ne posent pas le même genre de questions.

La première et la moins problématique de ces catégories est celle que constituent les genres qualifiés de savants, tels que les connaissent entre autres la plupart des civilisations d'Orient. D'origine souvent rituelle, ces musiques en ont parfois conservé certaines caractéristiques, notamment en ce qui concerne leur théorie, leur symbolisme, le comportement des participants et, bien sûr, leurs formes mélodico-rythmiques. Mais la plupart d'entre elles se sont progressivement détachées de cette fonction rituelle dans leur pays d'origine même où, sous l'effet des mutations sociales, elles sont devenues des expressions " artistiques» dans un sens comparable à celui que ce terme a acquis chez nous. La translation d'une musique rituelle en musique de cour destinée à une élite, puis en musique de concert en principe accessible à tous est ainsi un processus qui se rencontre de façon semblable en différentes cultures, et qui est marqué par un relâchement des liens entre le fait musical et son rôle social. Elle est même en quelque sorte la manifestation musicale du grand mouvement historique de laïcisation et de démocratisation caractéristique, à l'échelle mondiale, des temps modernes.

Rien ne s'oppose dès lors à l'exportation d'une telle musique, dans la mesure où elle est considérée plus comme un produit culturel que comme un agent cultuel. Les grands solistes de l'Inde et du Japon peuvent aujourd'hui être présentés dans des théâtres et des salles de concert à Paris, à Londres ou à San Francisco pratiquement de la même façon qu'à Delhi ou à Tokyo ; la relation entre artistes et auditoire s'y établit selon des codes semblables, avec le même type de conventions et dans un rapport de distanciation scènesalle absolument comparable.

17 Il est intéressant de relever qu'une grande partie des auditeurs autochtones de ces musiques, nés au sein de ces cultures orientales, ne sont aujourd'hui pas toujours nécessairement plus avertis que leurs nouveaux aficionados occidentaux. Leur participation est souvent devenue passive et conventionnelle - à l'instar de celle des abonnés aux saisons de musique classique en Europe - et motivée plus par des critères d'appartenance à une classe sociale que par des goûts personnels. De nombreux musiciens indiens sont à cet égard frappés, non seulement par l'intérêt sincère et profond, ainsi que par la qualité et l'intensité d'écoute que leur musique rencontre en Europe et en 
Amérique, mais aussi par la réelle connaissance de ses formes et de ses structures dont font preuve de nombreux amateurs étrangers, qui s'avèrent notamment capables d'en identifier les modes ou d'en suivre les rythmes complexes du début à la fin d'un récital.

Ces musiques se sont tout naturellement imposées en Occident de façon durable et elles comptent parmi celles qui y rencontrent l'adhésion la plus large. Le plaisir de la découverte de ces nouveaux univers sonores y est assorti pour le public de la jouissance d'arts extrêmement raffinés, à cet égard proches qualitativement de notre musique de chambre. Elles comportent en outre souvent une dimension de spontanéité et d'improvisation - généralement absente de cette dernière du fait de la fixation par écrit du répertoire classique occidental - qui, à cet égard, les rapprocherait plutôt du jazz. Les grands maîtres musiciens d'Orient sont aujourd'hui régulièrement sollicités par les organisateurs de concerts européens et appréciés par un public pratiquement acquis d'avance, enthousiaste et fervent.

19 Le domaine des musiques et des danses à caractère religieux et, d'une manière générale, de toute expression comportant une connotation sacrée ou une dimension rituelle attire également une large audience, dont les motivations sont d'ordre autant spirituel qu'artistique ou culturel dans le sens large. La présentation sur scène d'un ensemble de chant byzantin, d'un théatre mythologique hindou ou d'un chœur zoulou d'Afrique du Sud a ses amateurs, même si elle comporte en soi une certaine ambiguïté. La démarche suscite cependant des critiques, ses détracteurs y voyant une forme de simulacre, de profanation et d'induction au voyeurisme culturel. L'adoption des codes et des conventions propres au monde du spectacle - distanciation acteurs-public, éclairage artificiel, éventuelle sonorisation, billetterie, entracte, etc. - peut en outre constituer une barrière psychologique que certains spectateurs franchiront avec difficulté.

C'est ici la notion même de représentation qui est en cause et qui mérite effectivement d'être reconsidérée. La question est délicate et il convient de ne pas la contourner; on peut y répondre simplement que personne n'a jamais été contraint ni à se livrer à de telles exhibitions, ni, a fortiori, à y assister. Il apparaît en outre que des motivations très diverses animent les acteurs occasionnels de ces « spectacles rituels", parmi lesquelles le désir d'une action de type prosélyte est parfois lié à des soucis d'ordre politique ou économique. Significatif à cet égard est le cas de ces moines tibétains, désireux d'offrir la bénédiction du Dharma aux Occidentaux - qui, de leur point de vue, vivent aujourd'hui dans un monde dépourvu de spiritualité - tout en s'efforçant de réunir les fonds nécessaires à la reconstruction de leur monastère récemment détruit par un incendie, et en proclamant le droit de leur peuple à recouvrer l'autodétermination et l'indépendance de son pays. 
Fig. 1:Vrai rituel ou évocation: moines tibétains de l'ordre Gelug-pa en représentation à l'Auditoire Calvin, Genève

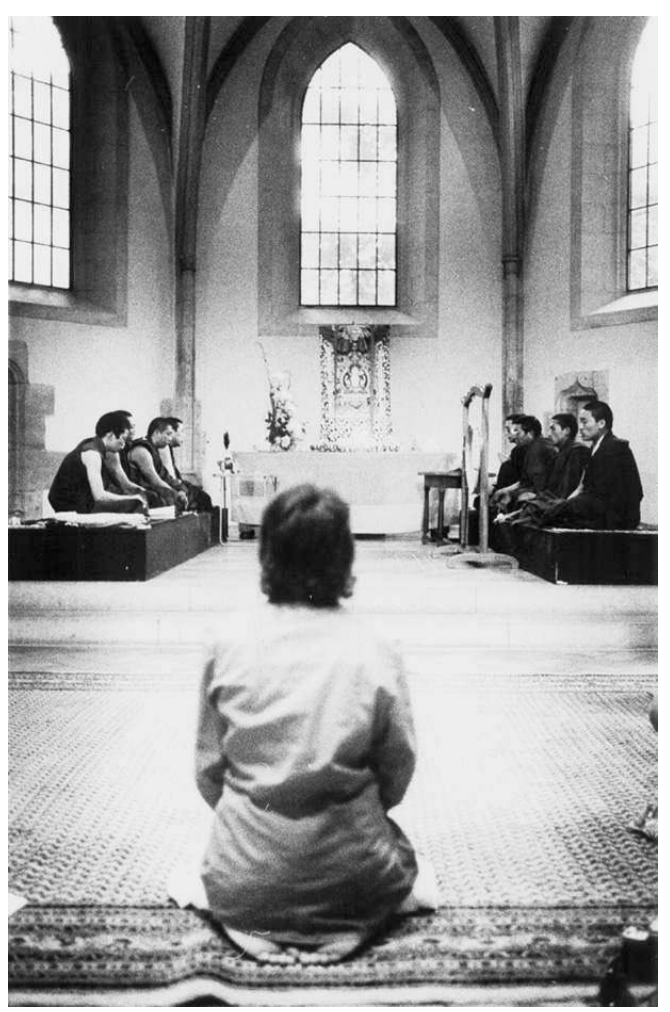

Photo: Yvan Devegney, 1984

21 Un autre exemple pourrait être celui d'un groupe de derviches orientaux, dont le maître souhaitait apparemment s'attirer de nouveaux disciples grâce à une série de manifestations organisées en Europe occidentale et aux Etats-Unis. A la fin de chaque cérémonie, il invitait le public à monter sur scène pour se faire initier à la pratique de certaines danses collectives. Peu à peu, ce qui devait être le témoignage d'une des pratiques les plus majestueuses de la mystique islamique a dégénéré en une sorte d'exhibition pénible et difficilement contrôlable, débordant largement les intentions de ses acteurs et, qui plus est, mal reçue par une grande partie du public. Rien n'est plus ennuyeux, en effet, pour un spectateur de type « culturel » que de voir une vingtaine de personnes monter sur scène pour se trémousser sans grâce sous prétexte de vivre une expérience spirituelle inédite. Sentant la tournure équivoque prise par les événements, le maitre en question résolut $1 / 2$ nalement de renoncer à cette forme de prosélytisme pour se consacrer à la formation de ses disciples.

Cette anecdote ne devrait cependant pas être prise pour plus que ce qu'elle est : un cas particulier de déviance. Mais elle est néanmoins significative de l'ambiguïté d'une démarche qui induirait plus à la curiosité du public, dans ce qu'elle peut avoir de malsain, qu'à sa formation et à son épanouissement. Il est avéré que l'attrait pour ces manifestations procède parfois d'une quête intérieure sincère, et que, confirmant une intuition préalable, le fait d'y assister peut provoquer chez certaines personnes une sorte d'étincelle, de déclic révélateur, dont les conséquences sont, en tout état de cause, difficilement analysables. 
Toujours dans le cadre du soufisme oriental, le cas du chanteur de qawwâlî pakistanais Nusrat Fateh Ali Khan est significatif d'un autre type de phénomène: l'accès au «star system » d'un artiste, certes d'un talent et d'un charisme exceptionnels, mais dont la vocation première était, pour le moins, d'un autre ordre, à savoir de chanter les louanges du Prophète et des saints de l'islam dans l'enceinte des sanctuaires musulmans. Rendu mondialement célèbre par ses innombrables disques compacts ${ }^{7}$, dont les ventes sont régulièrement relancées par de fréquentes tournées de concerts en Occident, Nusrat a été le principal responsable de la mutation d'une tradition musicale religieuse à caractère ésotérique en un produit de consommation de masse. Apparemment sans effets sur ses motivations et sur sa foi islamique, ces développements inattendus de sa carrière représenteraient pour lui tout au plus "just a little experiment", si l'on en croit un entretien accordé au New York Times en 1993 (cit. in Sakata 1994: 96). Pourtant, par rapport à la tradition dont il est issu, Nusrat représente bien un phénomène hors du commun, non seulement par l'aisance déconcertante de son talent, mais aussi par les innovations stylistiques qu'il a introduites ${ }^{8}$ et, tout autant, par l'utilisation mercantile qui a été faite de sa musique - avec son total consentement, précisons-le9

Une autre catégorie musicale importante et fréquemment présentée dans nos théâtres est celle des expressions dites populaires. Parmi celles-ci, les musiques de fête occupent une place centrale. Les fêtes «se caractérisent par des propriétés contradictoires, relève Bernard Lortat-Jacob; [...] elles ont une fonction à la fois conservatrice et transformatrice » (1994: 7). La fête représente en effet souvent le lieu de jonction entre les domaines du sacré et du profane; échappant aux conditions cycliques du temps ordinaire, elle opère dans certaines civilisations à la manière d'une évocation de l'Age d'Or ; ailleurs, elle apparaît comme une célébration collective destinée à raffermir tant les liens sociaux que ceux rattachant la communauté concernée à ses références spirituelles ; ailleurs encore - notamment dans les fêtes de type carnavalesque - comme une inversion temporaire et "compensatoire " des valeurs établies. Associée ou non à la danse, la musique qui anime la fête est, de manière générale, de nature dionysiaque, par opposition à celle des genres savants évoqués précédemment, qui est plutôt apollinienne dans ses principes, ses critères et ses conditions de jeu ${ }^{10}$. 
Fig. 2a-b:La vie d'artiste: le joueur de didjeridu Richard Walley, «aborigène» australien de l'ethnie Yungar, et ses amis à Genève:

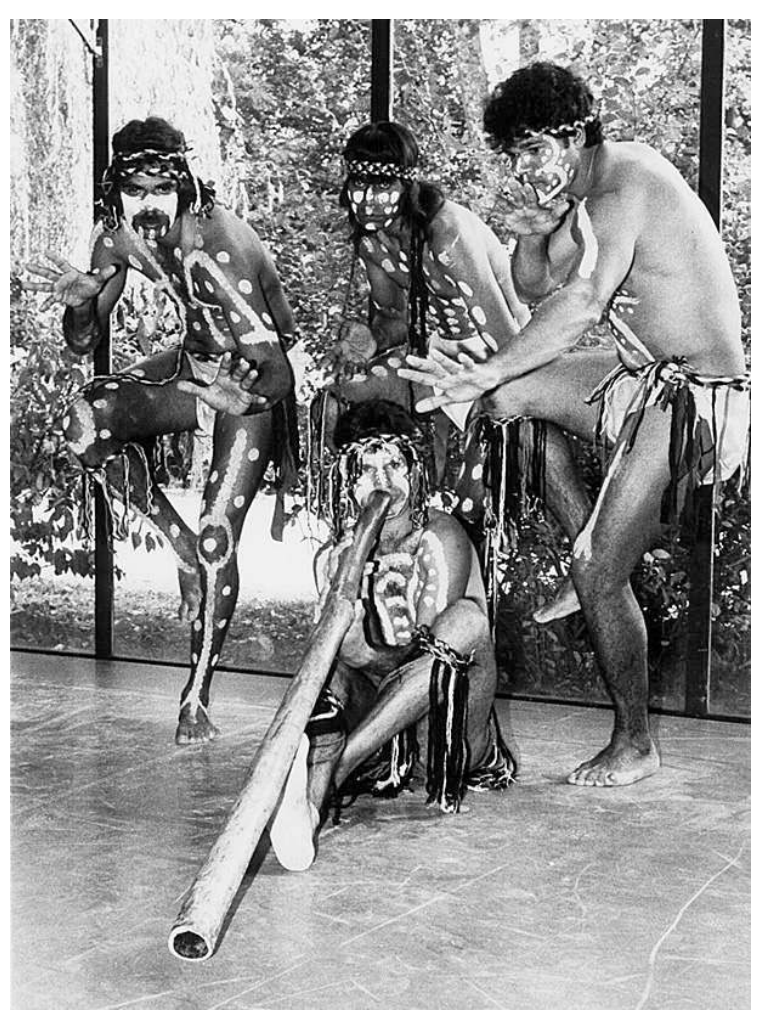

(a) en animation scolaire dans une salle de gymnastique;

(b) en enregistrement à la Maison de la Radio.

Photos: Jacques Berthet, 1985

Les musiques de fête de nombreuses régions du monde sont aujourd'hui largement appréciées hors de leurs frontières, tant il est vrai qu'elles paraissent les plus directement accessibles à tout un chacun, même si les codes auxquels elles font appel ne sont pas toujours perçus dans leur pleine mesure. Elles s'adapteront cependant mieux à des événements pouvant rappeler leurs circonstances de jeu traditionnelles qu'aux concerts conventionnels en salle. Même si des ensembles comme les Musiciens du Nil, les orchestres tsiganes roumains ou turcs, les fanfares de toute provenance ou les innombrables groupes de percussions africains et afro-américains actifs en Europe sont capables de soulever l'enthousiasme en situation de concert - une situation anormale pour ces musiciens -, ils ne seront cependant jamais plus à l'aise, à l'étranger, que lors de festivals en plein air, joyeuses célébrations païennes et pluriculturelles, auxquelles ils s'adaptent avec un bonheur évident.

Il existe enfin une catégorie musicale particulièrement « exotique » et souvent fragile hors de ses limites normales d'expansion: celle que constituent les expressions des peuples volontiers considérés comme «primitifs». Malgré ses connotations ethnocentristes et évolutionnistes, ce terme sert encore trop souvent à cataloguer dans leur ensemble des nations autochtones - dans le sens défini par les organisations internationales non gouvernementales concernées par leurs problèmes - désignées par des appellations telles qu'Aborigènes, Bochimans, Pygmées ou Esquimaux. Il faut cependant rappeler que, tout autant que celle de "primitifs ", ces dénominations sont 
toutes d'origine coloniale et que les peuples concernés les contestent dans leur ensemble en raison des relents ségrégationnistes qu'elles dégagent.

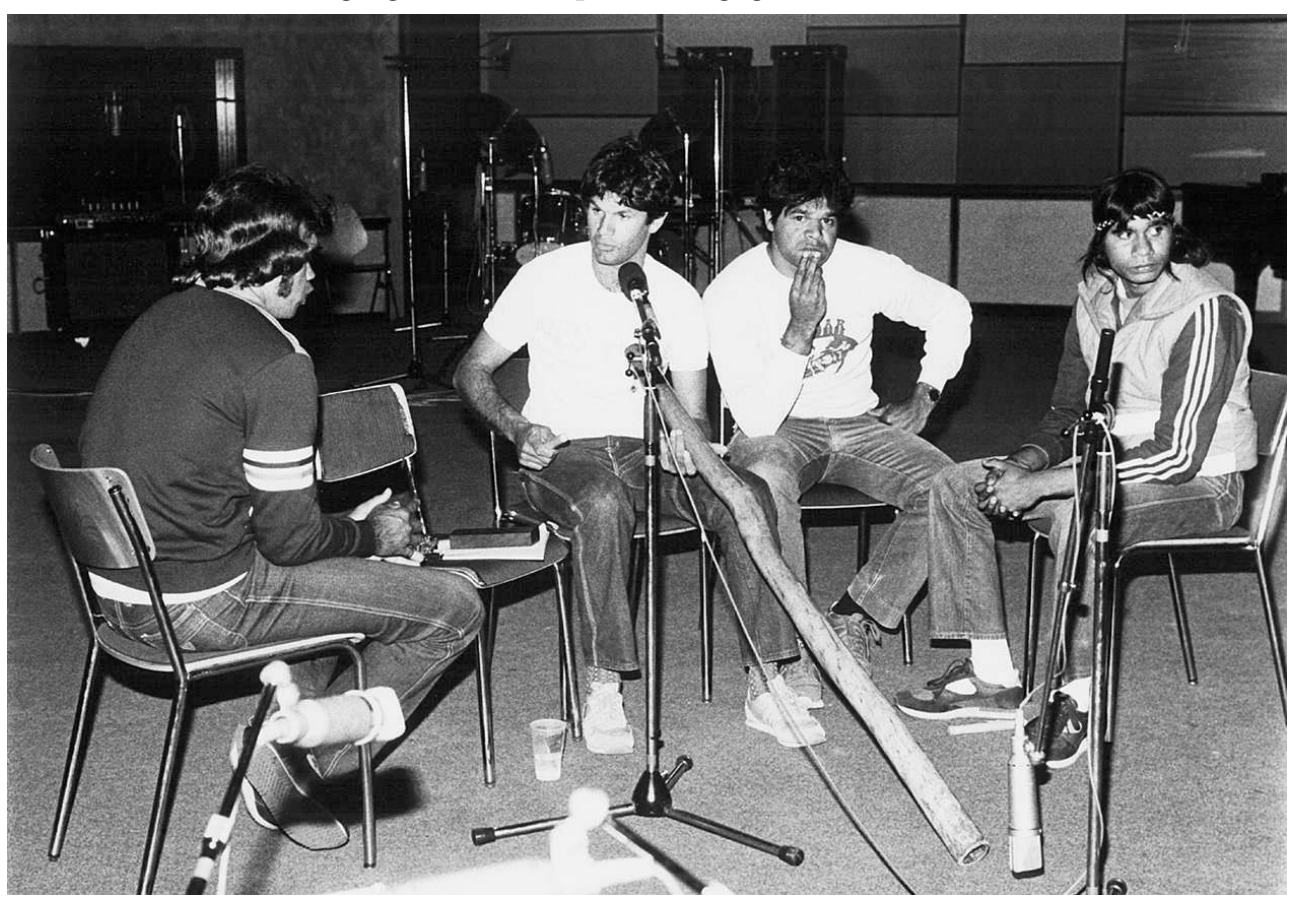

Comment pouvons-nous appréhender hors contexte l'univers sonore de peuples pour lesquels la musique et la danse - ou ce que nous considérons comme tels, ces concepts n'étant pas toujours pertinents pour leurs producteurs - n'ont d'existence que dans le cadre d'une fonction précise à laquelle leur expression est totalement subordonnée ? La pratique musicale y répond en effet à des critères totalement différents des nôtres, et sa dimension esthétique - pour nous la plus immédiatement perceptible - ne peut être judicieusement évaluée qu'à partir de ses propres codes d'appréciation et, qui plus est, dans son environnement traditionnel et à la lumière de cet environnement.

Un exemple significatif à cet égard a été fourni en 1988 par l'invitation en Europe d'une délégation des peuples Melpa et Huli, deux ethnies papoues des Highlands de Papouasie Nouvelle Guinée, dans le cadre d'un festival sur les musiques et danses du Pacifique. La première représentation de ces artistes occasionnels fut marquée par quelques incidents assez cocasses. Parés de leurs superbes atours de cérémonie, et notamment de coiffes de plumes et de peintures faciales aux couleurs très vives, les danseurs firent leur entrée sur le plateau d'un pas hésitant, ne sachant apparemment pas très bien ce qu'on attendait d'eux. Une fois sur scène, ils furent stupéfaits de voir que des centaines de personnes étaient confortablement assises face à eux dans la pénombre, figées dans un silence opaque. Interloqués, ils ne savaient pas comment réagir, manifestement mal préparés aux exigences de la scène. Après un bref conciliabule, ils éclatèrent de rire, désignant du doigt ces spectateurs si attentifs, mais incompréhensibles dans leur absence de réactions. La glace était rompue, et leur hilarité communicative fut bientôt partagée par le public, sensible au comique de cette situation peu ordinaire. La performance put alors commencer et se dérouler à peu près normalement, encore que de façon assez décousue, faute des repères orientant traditionnellement la succession de ses phases. Le «spectacle» fut en définitive bien reçu par une grande partie du public, sensible à la nature de ce " premier contact » d'un genre nouveau, alors qu'il suscita la réprobation, voire la colère de certaines personnes, convaincues de l'indécence d'une telle démarche. 
29 L'inclusion de telles prestations dans des festivals ou des saisons de concerts donne évidemment à réfléchir. Que sommes-nous en effet à même d'apprécier de ces cultures lorsque nous n'avons accès qu'à une de leurs composantes, arbitrairement privilégiée et extraite de ses structures de référence ? Et que peut apporter un tel simulacre - qui est inévitablement aussi une réduction de la réalité - à ceux qui ont accepté de s'y prêter? Ont-ils les moyens d'évaluer l'image qu'ils fournissent d'eux-mêmes? Quels en sont les enjeux pour eux? Ont-ils enfin la possibilité d'en mesurer les incidences sur leur perception ultérieure de leur propre culture et de son système de valeurs?

$\mathrm{Du}$ point de vue de l'organisateur, il serait discutable d'exclure arbitrairement de sa programmation certains genres, voire l'ensemble des manifestations de certaines cultures, sous prétexte que leur présentation aurait un caractère «douteux » ou qu'elle n'est pas suffisamment «spectaculaire». Il doit cependant se méfier d'une forme de paternalisme tiers-mondiste consistant à accumuler les programmes exotiques comme d'autres collectionnent les papillons, sans tenir compte des problèmes spécifiques posés par chaque cas.

31 Lorsqu'elles ont lieu, les prestations de tels groupes provoquent d'ailleurs d'interminables débats parmi le public : les uns en ressortent mal à l'aise, se sentant voyeurs malgré eux, estimant à juste titre qu'il manquait quelque chose d'essentiel à ce à quoi ils viennent d'assister, que «ce ne sera jamais comme là-bas ", et qu'en définitive la démarche est en soi perverse; d'autres, en revanche, y adhèrent sans restriction, considérant que leur participation à de tels événements est pour eux un privilège rare, qu'elle leur a apporté un moment de grâce incomparable ou qu'elle a contribué à remettre en cause certains de leurs préjugés culturels.

Ces deux positions sont chacune respectables et partiellement fondées, même si elles sont inconciliables. Sans qu'il soit possible de trancher de façon péremptoire, elles montrent en tout cas que le responsable culturel confronté à ces questions s'engage autant sur le plan de l'éthique que sur celui de l'ouverture artistique. La préparation psychologique et l'accueil de tels groupes, la manière de les présenter et le lieu choisi à cet effet revêtent de ce fait une importance toute particulière, et il est également capital de fournir aux spectateurs qui le souhaitent une documentation aussi claire que possible sur les tenants et aboutissants de ce qui leur est proposé comme spectacle.

\section{La vie d'artiste ou les exigences d'un nouveau statut}

33 La scène est le lieu de la représentation à un triple titre : d'abord simplement parce qu'elle est une occasion de jeu (la musique comme art), puis en tant que processus évocatoire d'une réalité (la musique « en situation»), et enfin comme signe d'une totalité qui inclut la musique et qu'elle manifeste (la société dont elle provient et ses valeurs). Elle constitue pour les musiciens qui y sont présentés un débouché nouveau, souvent inattendu et, qui plus est, lucratif, ce qui influence forcément leur appréciation. Le concert présente en outre pour eux une occasion souvent unique de voyager à l'étranger et, éventuellement, d'être ensuite valorisés au sein de leur propre communauté, voire par les autorités de leur pays ${ }^{11}$. En privilégiant la dimension esthétique - sonore et visuelle $\mathrm{du}$ fait musical et en faisant l'impasse sur ce qui constitue son environnement ordinaire, le phénomène a imposé à l'ensemble des musiciens qui $\mathrm{y}$ accèdent des exigences nouvelles, dont la première se rapporte au fait qu'ils y sont considérés comme des « artistes » et non, ainsi que c'est souvent le cas dans leur propre contexte social, comme 
des acteurs parmi d'autres d'un événement auquel chacun participe à sa façon, selon le rôle qui lui est assigné ou qu'il se choisit.

La configuration de nos théâtres, de nos salles de concerts ou de nos scènes de festivals impose en outre un rapport frontal stéréotypé qui crée une distance irréductible entre l'artiste ou les artistes, éclairés par les feux de la rampe, et le public, confiné dans l'obscurité et censé demeurer passif, silencieux et figé dans une attitude de réceptivité quasi dévote, sauf aux moments où son appréciation doit nécessairement se manifester avec fracas par des applaudissements plus ou moins longs et fournis selon le degré de son enthousiasme.

Ce statut d'artistes implique que les musiciens se plient aux codes du concert, notamment en ce qui concerne les questions de style, de durée et de qualité formelle de leur prestation, faute de quoi leur message ne sera pas reçu dans sa pleine mesure. Un concert est ainsi en principe censé durer d'une heure et demie à deux heures, dans certains lieux avec un entracte imposé à mi-parcours. La prestation doit donc impérieusement s'adapter à ces exigences; si elle est plus courte, le public aura l'impression de ne pas en avoir eu pour son argent, et si elle est plus longue, la salle se videra progressivement pour diverses raisons : les auditeurs commenceront à se lasser, il ne leur faut pas manquer le dernier métro, ils travaillent le lendemain...

La question de la durée affecte également chaque pièce du programme et, là aussi, il convient de tenir compte de critères dépendant du «seuil de tolérance» du public moyen. Si l'interprétation d'un râga par un grand maître indien se développe en moins de quarante-cinq minutes, il se verra accusé d'adapter sa musique aux goûts de l'Occident ; si, en revanche, elle dépasse une heure et demie, une bonne partie de l'auditoire sera incapable de maintenir sa concentration aussi longtemps.

Dès lors qu'il accepte les conventions du concert, le musicien assume implicitement qu'étant en représentation, il ne peut pas exiger que les choses se passent sur scène comme chez lui. Comment, en effet, un barde d'Asie centrale ou un conteur africain pourrait-il par exemple espérer maintenir l'attention d'un public ne comprenant pas sa langue durant l'intégrale d'un chant épique long de trois heures? Il est amené à faire une série de concessions, auxquelles il se plie d'ailleurs généralement de bonne grâce, pour peu qu'il ait un minimum de sens psychologique et de connaissance préalable des réalités de la scène.

Les techniques vocales et instrumentales devront en principe être conformes à nos critères d'authenticité, ne donnant autant que possible aucun signe d'occidentalisation ; comme celui de la musique "ancienne» européenne, le public des musiques traditionnelles est particulièrement sensible aux timbres de la musique et à la pureté des sons. Une voix comportant un vibrato excessif sera considérée comme acculturée et esthétiquement non conforme ; un orchestre ne devra en aucun cas se livrer à la moindre tentative d'harmonisation "modernisante», ni comporter d'instruments d'origine étrangère, surtout pas d'instruments électroniques. D'une manière générale, le concert de musique traditionnelle se doit d'être culturally correct, faute de quoi il s'expose à la critique la plus vive.

Conscients de ces goûts du public, qu'ils partagent d'ailleurs pour la plupart, les organisateurs s'efforcent de présenter des programmes répondant à ces critères, parfois avec un remarquable discernement esthétique, parfois au contraire avec une forme de purisme confinant à l'absurde. Dans un article pour la revue Trad Magazine, Henri Lecomte 
a ainsi eu l'occasion de s'en prendre à un prestigieux responsable artistique qui, négociant la venue en France du grand chanteur tadjik Adineh Hashemov et de son ensemble, l'avait « interdit d'accordéon » sous prétexte que cet instrument n'était pas originaire du Tadjikistan et qu'il ne permettait pas de produire les subtiles ornementations mélodiques ni les intervalles microtonaux présumés de cette musique (Lecomte $1992: 28)^{12}$.

40 Cette anecdote est significative d'un certain état d'esprit prévalant parfois dans le milieu, qui a tendance à appliquer des critères relevant proprement de l'intégrisme culturel à la sélection des artistes et des programmes, en particulier lorsqu'il s'agit de cultures non occidentales. Un instrumentiste oriental se laissant aller à un excès d'arpèges ou de tierces parallèles dans ses improvisations sera rejeté comme un musicien au goût douteux. Il est possible que, dans son pays, il n'aurait jamais joué de cette façon ; mais son intention n'était peut-être que de plaire à ses auditeurs - suprême marque de corruption pour la fraction doctrinaire du public - et de se rapprocher d'eux en leur montrant, certes de façon naïve et inappropriée, qu'il connaissait aussi la musique occidentale ${ }^{13}$. Je me souviens à ce propos des soupirs consternés d'une partie du public assistant à un concert du maitre du sarod indien Ali Akbar Khan quand ce dernier jugea bon d'inclure des extraits de "Greensleaves » ou d' "Au clair de la lune» dans ses improvisations, par ailleurs d'une beauté fulgurante. Ce qui n'était pour lui qu'un trait d'humour passager, un clin d'œil malicieux, fut perçu par une partie du public comme un acte iconoclaste intolérable, et les commentaires entendus dans le foyer après le concert tournaient essentiellement autour de l'influence jugée néfaste de sa résidence californienne sur le génie de cet artiste, sans parler de celle, présumée, de l'alcool et des femmes...

Une autre donnée du concert de musique traditionnelle suscite de nombreux débats au sein du public: le problème de l'amplification sonore. Pour certains auditeurs, celle-ci représente le mal absolu car, de manière pour eux évidente, il s'agit d'une forme de manipulation et d'acculturation indigne des musiques qu'elle affecte. Ce que ces gens ignorent pour la plupart, c'est que ce sont les musiciens eux-mêmes qui exigent l'usage de microphones, soit que ceux-ci représentent pour eux une sorte de consécration technologique, soit que l'utilisation en soit depuis longtemps généralisée chez eux.

42 Le récent développement d'une musique comme celle de la civilisation mandingue d'Afrique de l'Ouest n'a par exemple été rendu possible que grâce à l'apport de la sonorisation, lequel permet de mêler au sein d'un orchestre des instruments autrefois incompatibles du fait de leur différence de volume sonore. L'ensemble "classique contemporain » des griots d'Afrique occidentale, auquel nous faisions allusion en début d'article, comporte ainsi aujourd'hui presque invariablement les instruments suivants : la harpe-luth kora, le luth ngoni et/ou la guitare européenne à cordes métalliques, la flûte traversière flé et le balafon, tous des instruments à sons relativement doux, auxquels sont aujourd'hui associés divers tambours extrêmement puissants, en particulier le djembé. Faudrait-il alors interdire à cette musique d'évoluer sous prétexte que son développement implique l'usage d'une technologie importée?

Quant à la musique "savante» de l'Inde, pourtant par excellence une musique de chambre, je n'en ai rencontré en vingt ans qu'un seul interprète préférant jouer sans amplification; encore s'agissait-il d'un musicien relativement atypique, joueur de vînâ depuis longtemps émigré en France, et dont les conceptions avaient été fortement influencées par celles d'amis impliqués dans le courant de la musique ancienne européenne. Sinon, quelles que soient les dimensions et l'acoustique de la salle, tous 
exigent des micros. Les frères Dagar, grands spécialistes du chant dhrupad, affirmaient même que l'amplification leur avait permis d'affiner considérablement l'ornementation vocale, du fait qu'ainsi « tout s'entend », et qu'il ne leur était dès lors plus nécessaire de continuellement forcer leur voixdans les parties rythmées de leurs récitals pour éviter qu'elle soit couverte par l'accompagnement du tambour pakhavaj. Voici un autre cas de conscience pour nos censeurs nostalgiques!

Fig. 3: La voix portée au loin: les frères Zahiruddin et Faiyazuddin Dagar sur scène à Paris

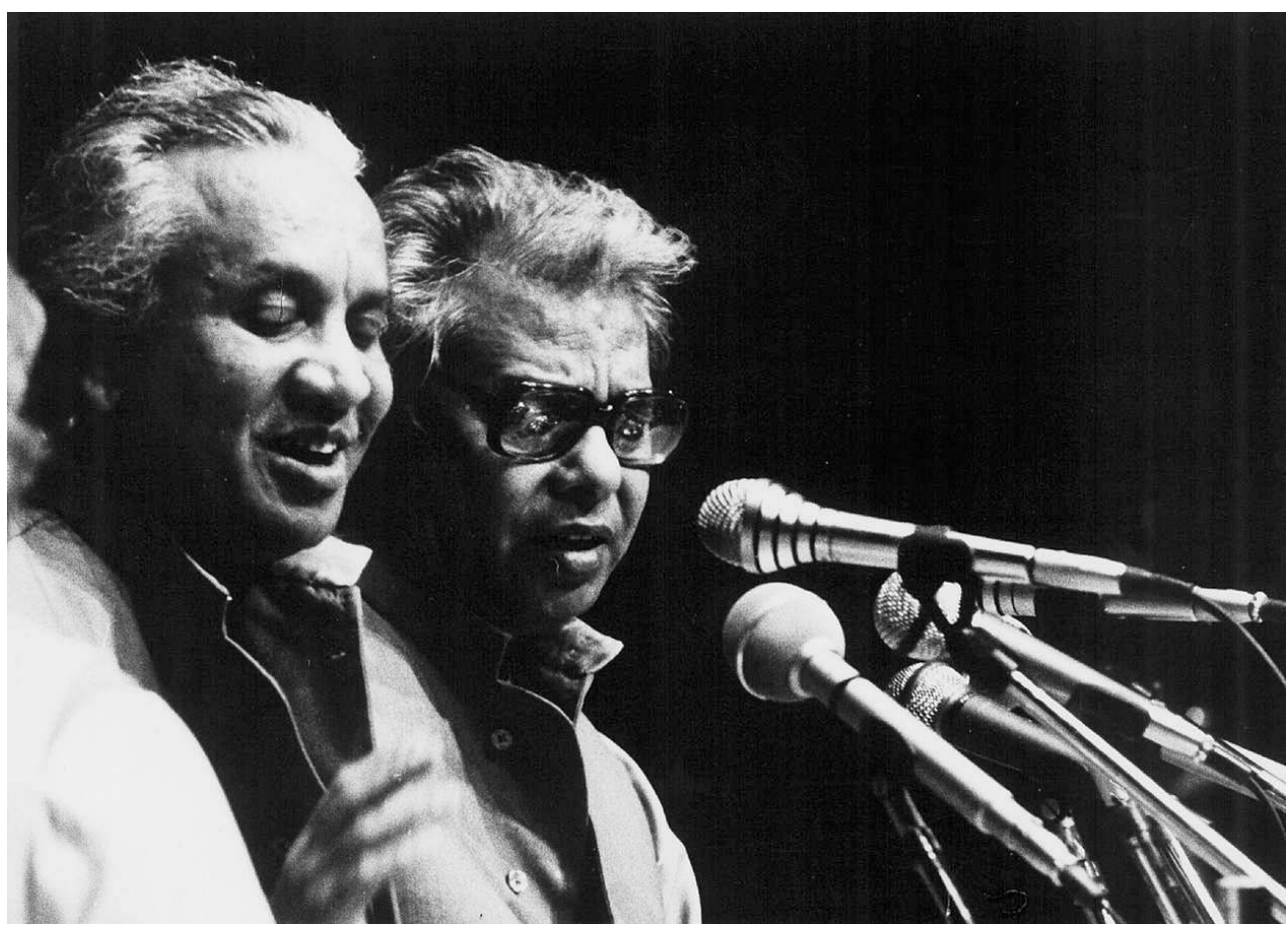

Photo: Dhrupad Society, Paris, 1987

Il est aussi souhaité que les costumes de scène des musiciens correspondent à l'image escomptée de leur culture - une image nécessairement idéalisée -, faute de quoi ils risquent de ne pas passer pour des interprètes authentiques. Il arrive ainsi fréquemment que des musiciens se voient imposer pour une tournée le port de vêtements traditionnels - voire, si ceux-ci sont devenu obsolètes, d'« habits d'époque » reconstitués pour les besoin de la cause. Peu importe si, chez eux, ils se produisent aujourd'hui en frac ou en tenue ordinaire "de ville » : la scène étant le lieu de la représentation - et donc de l'idéalisation -, il s'agit d'en ménager les codes visuels au même titre que les conventions sonores. Le grand luthiste irakien Munir Bashir l'a bien compris, lui qui, après avoir débuté sa carrière internationale en complet-cravate - afin probablement de se faire reconnaître comme un artiste "classique " et respectable en tant que tel - arbore aujourd'hui, lorsqu'il se produit en Occident, de splendides gandouras censées correspondre à l'esthétique sonore de sa musique. 
Fig. 4:Tenue de scène: le joueur de darbuka Güngör Hosses, Tsigane d'Istanbul, en concert à Amsterdam

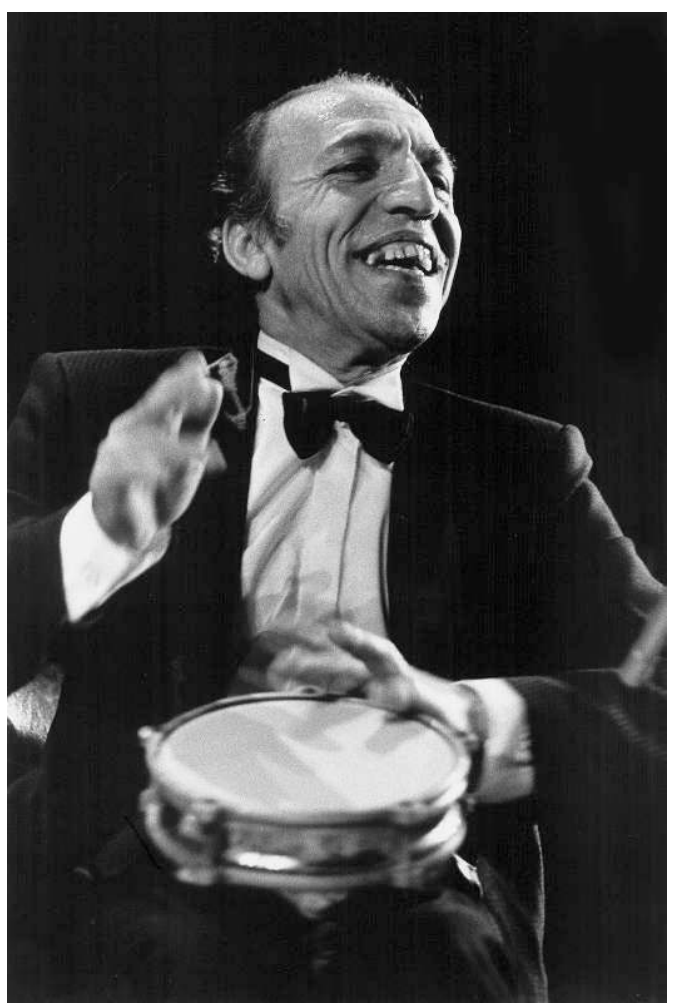

Photo: Ton Verhees, 1990

Quant au remarquable ensemble de xylophones timbila des Chopi du Mozambique, mené par le maître Venancio Mbande, l'authenticité - encore elle - de sa prestation a récemment été mise en doute par certains spectateurs du fait que les musiciens et les danseurs se produisaient sur scène vêtus de maillots de coton et de survêtements de nylon aux couleurs jugées agressives - en fait celles du drapeau national! Ce que ces donneurs de leçons ne savaient manifestement pas, c'est que, depuis plusieurs générations, les membres de ce groupe - tous des amateurs, dans le meilleur sens du terme - sont recrutés parmi les mineurs mozambicains émigrés en Afrique du Sud, et qu'il serait pour eux tout aussi incongru de se produire vêtus de pagnes qu'à un interprète actuel de Mozart de porter sur scène un jabot de dentelle et une perruque poudrée.

La reconstitution sur scène d'une cérémonie - noce avec fausse mariée, fête villageoise avec réjouissances simulées, rituel avec transe feinte, etc. - est en revanche souvent mal perçue, nous l'avons vu plus haut. Une partie du public a l'impression désagréable de se trouver en situation de voyeur, se sentant exclue du processus se déroulant devant ses yeux, démunie de tout moyen d'y participer; sa réaction est alors souvent qu'une telle représentation n'est pas authentique et qu'il s'agit de folklore arrangé et non plus d'une prestation à caractère traditionnel - ce qui est d'ailleurs souvent exact, quelles que soient les intentions des artistes et de l'« arrangeur » du spectacle.

On peut mentionner à ce propos l'exemple d'une troupe réunissant une douzaine des meilleurs danseurs et chanteurs Lakota Sioux récemment fondée autour d'un chef charismatique dans l'intention louable de faire découvrir leurs coutumes aux Européens. 
Soigneusement préparé avec l'assistance de spécialistes étrangers, le programme visait à présenter un maximum de danses pratiquées lors des pow-wow traditionnels. Mais, malgré des chants, des danses et des costumes d'une esthétique irréprochable, « le courant ne passait pas » entre ces remarquables défenseurs des valeurs culturelles amérindiennes et leur public d'un soir, pourtant manifestement acquis d'avance à leur cause. La raison en était simple : les danses étaient chacune réduites à une brève démonstration de deux ou trois minutes, ne laissant le temps ni aux interprètes d'en développer le caractère, ni à leurs spectateurs d'entrer dans le monde si particulier évoqué par chacune d'entre elles. Le programme était en outre incessamment entrecoupé de commentaires oiseux d'un présentateur, moins destinés à informer le public qu'à, semble-t-il, détendre l'atmosphère. La qualité intrinsèque des artistes, l'authenticité de leur répertoire et la pureté de leurs intentions ne furent ainsi pas suffisants pour garantir le succès d'une telle entreprise; un minimum de connaissance des lois de la scène et de la psychologie du public européen - souvent peu sensible aux vertus du show à l'américaine - eut également été nécessaire pour éviter ce genre de maladresse. Continuellement pressés par le temps, les danseurs n'avaient pas l'occasion de s'investir dans leurs danses ; ils ne pouvaient que les montrer, et cela se sentait !

La représentation théâtrale d'un rituel n'exclut pourtant pas en soi la possibilité d'un réel investissement de ses acteurs, ni de l'efficacité de la cérémonie en jeu. Des derviches turcs m'ont dit avoir ressenti sur scène des états d'extase absolument comparables à ceux qu'ils connaissent habituellement dans leur tekké d'Istanbul, portés notamment par le sentiment de ferveur collective dégagé par le public. Dans le même ordre d'idée, une prêtresse du candomblé afro-brésilien, invitée avec un groupe d'adeptes à présenter en Europe une évocation stylisée d'un rituel, m'a dit avoir feint la transe pendant tous les spectacles de la tournée, mais qu'à Genève - où nous l'accueillions -, elle était réellement tombée en transe et que, pour la première fois depuis qu'elle avait quitté le Brésil, les orixás s'étaient manifestés à elle ce soir-là. Elle en paraissait elle-même bouleversée, et cette expérience semble même avoir remis en question certaines de ses idées sur les conditions de la transe.

Fig. 5:Extase réelle ou extase feinte: les derviches tourneurs Mevlevi dansant pour les touristes dans la grande halle des sports de Konya (Turquie)

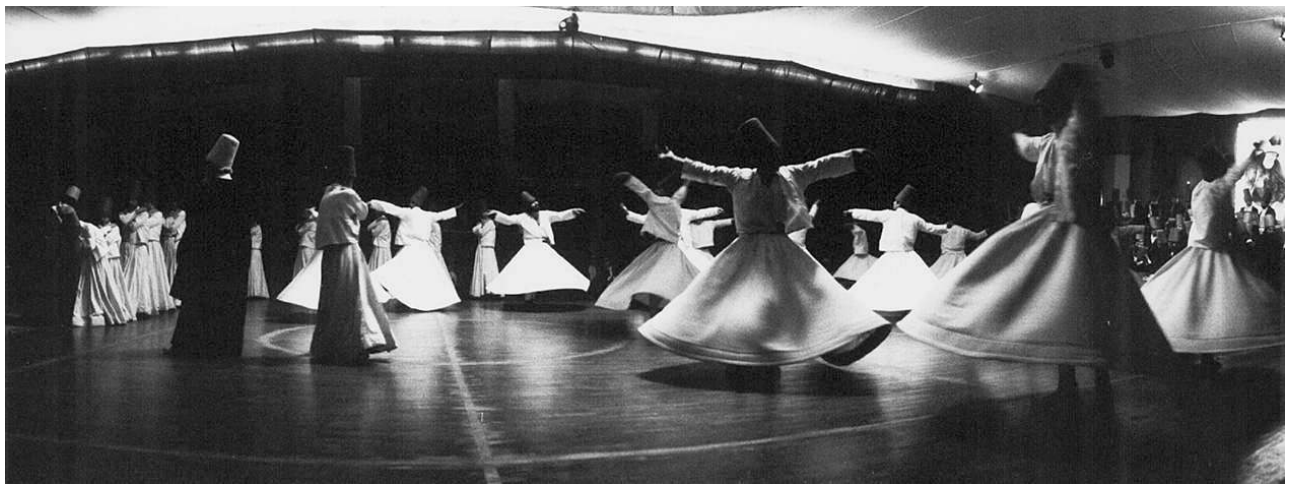

Photo: Jacques Bétant, 1982

D'une manière générale, les musiciens et les danseurs s'adaptent sans problème aux exigences de la scène, faisant même preuve à cet égard d'une remarquable ingéniosité ; les rares dérapages dans ce domaine semblent plus venir de maladresses de promoteurs ou d'accompagnateurs que d'erreurs de jugement des interprètes eux-mêmes. La plupart 
de ceux-ci sont parfaitement conscients des enjeux en présence et des concessions auxquelles il leur faut parfois se plier s'ils veulent faire apprécier leur art à sa juste valeur; ils y consentent volontiers, souvent même avec un certain humour de circonstance, ce qui ne signifie pas qu'ils soient tous prêts à accepter n'importe quel compromis contraire à leurs principes.

Mais ces nouveaux débouchés pour les musiques traditionnelles du monde ont forcément une influence sur les modalités de leur expression. Les conditions de jeu sont différentes de celles rencontrées dans leur contexte habituel, en particulier en ce qui concerne la nature des relations entre "musiquants » et " musiqués ${ }^{14}$. Faute de pouvoir analyser ici tout l'éventail des changements occasionnés par ce transfert, relevons quelques tendances fréquentes. On remarque en premier lieu la propension à un certain purisme de circonstance, à la recherche d'une image visuelle et sonore " correcte ", conforme aux règles du genre; la démarche est d'ailleurs semblable à celle qui est appliquée dans l'interprétation contemporaine des musiques anciennes européennes - avec lesquelles plus d'une musique traditionnelle partage de nombreux traits, nous l'avons déjà vu ${ }^{15}$.

51 L'image que les musiciens andins entendent donner de leur culture à l'étranger s'est par exemple modifiée au cours des trente ou quarante dernières années. Dans un premier temps, on a assisté à un déferlement d'orchestres pratiquant un folklore "rénové ", métissé, virtuose et sagement arrangé, dont les modèles étaient des groupes tels que Los Incas ou Los Calchakis; depuis le début des années quatre-vingt, on observe en revanche un retour en force d'ensembles calqués sur ceux des communautés aymara de l'Altiplano, pour lesquels l'absence d'instruments à cordes et le port de la tresse et du poncho sont les signes d'une revendication identitaire.

Une autre occurence se rapporte au niveau d'exigence du concert sur le plan de la performance, un niveau proportionnel au degré de familiarité du public avec le genre présenté. Les guitaristes flamenco se doivent par exemple de tenir compte des prouesses de Paco de Lucía, les joueurs de sitar indien de celles de Ravi Shankar ou de Vilayat Khan, et les jembéfola africains de cellesd'Adama Dramé ou des Percussions de Guinée, car il savent qu'ils seront jugés par rapport à ces critères d'excellence. Il en résulte une nouvelle sorte d'émulation, poussant la plupart des interprètes à sans cesse repousser les limites de leurs possibilités techniques. Le résultat est effectivement que leur virtuosité s'est considérablement accrue ces dernières années, parfois jusqu'à des sommets inouïs. Mais de nombreux connaisseurs s'accordent à déplorer la perte de musicalité qui en découle pour ces athlètes de la musique, motivés plus par son aspect quantitatif calculable en nombre de notes allignées à la minute - que par la qualité de l'expression et de l'émotion qui s'en dégage.

53 Comme dans n'importe quel autre domaine, la loi de la concurrence joue désormais, imposant ses règles à ceux qui s'y prêtent. L'internationalisation du marché représente aujourd'hui un nouveau défi pour des musiciens qui ne peuvent plus, comme par le passé, tabler sur le soutien de structures sociales qui leur garantissaient souvent bien-être et respectabilité au sein de leur propre culture. Fragile, leur statut est constamment remis en question par les courants de la mode et les enjeux qu'ils représentent. Mais en définitive, n'est-ce pas précisément là, dans cette preuve manifeste de liberté, que représente le «bon usage » des contraintes, que réside la nature du véritable artiste? 


\section{L'art de bien entendre : esquisse d'une typologie de l'auditeur}

54 «La musique est humaine à la condition d'admettre que le son n'est pas seulement organisé et pensé par celui qui le produit, mais aussi par celui qui le perçoit ", écrit JeanJacques Nattiez $(1987: 86)$ en réponse à Blacking ${ }^{16}$. Après avoir examiné l'influence de la situation nouvelle que constitue le concert sur les paramètres de l'émission de musiques qui n'y sont a priori pas destinées, il convient donc, dans ce cas particulier, de confronter ces paramètres à ceux, complémentaires, de la réception musicale. De manière générale, celle-ci est conditionnée par de nombreux facteurs, dépendant tant de la personnalité du « récepteur » que de celle de l'« émetteur » et des circonstances dans lesquelles s'établit leur relation. Il n'y a pas de relation biunivoque entre ce que Nattiez appelle les « stratégies de production (poïétiques) » et les « stratégies de perception (esthésiques) » ( ibid. : 94-95), et l'interdépendance de ces facteurs se manifeste sous forme d'un réseau complexe de correspondances, individuelles et collectives entre «musiquants" (émetteurs) et « musiqués » (récepteurs). Plusieurs personnes placées simultanément face au même événement ou objet sonore - musical ou non - le percevront ainsi de façon différente en fonction d'un éventail de déterminants personnels, relatifs à l'auditeur en tant que sujet et à ce que Pierre Schaeffer appelle ses « intentions de perception » (1966: 114). Parmi celles-ci, il distingue «deux couples de tendances caractéristiques de l'écoute: il s'agit d'abord d'opposer l'écoute naturelle à l'écoute culturelle, puis de comparer l'écoute banale à l'écoute spécialisée ou praticienne » (ibid.: 120). Une autre distinction, à mon sens plus pertinente, consisterait à répartir tout d'abord les auditeurs en deux catégories relatives à leur attitude face la musique : les auditeurs distanciés, dont l'appréciation passe avant tout par une approche analytique et, en principe, objective de ce qu'ils entendent, et les auditeurs participants, dont l'écoute, de nature plus synthétique, est nourrie par des aspirations d'ordre essentiellement subjectif et émotionnel; les premiers sont surtout sensibles aux formes et aux structures de la musique, les seconds davantage à son contenu et à ses effets.

Dans toute performance musicale en présence de public, une relation de réciprocité de sujet à sujet s'établit nécessairement entre musiciens et auditeurs, dans la mesure où la nature de la réception conditionne au plus haut point celle de l'émission. Consciemment ou non, un musicien joue toujours pour ceux qui l'écoutent; son interprétation est déterminée par la conjonction d'un ensemble de facteurs circonstanciels : ses propres dispositions du moment et les conditions psychologiques créées par la circonstance dans laquelle il se produit, certes, mais tout autant la réceptivité de son public et les réactions - manifestées ou tues, mais toujours sensibles - que la musique produit en lui. Sauf en cas de solo, un musicien est aussi en interaction intime avec ses partenaires, dont la complicité exerce une influence prépondérante sur son jeu individuel ; quant à l'auditeur, sa perception est également modifiée par celle des personnes qui l'entourent. Trois ordres relationnels interdépendants se manifestent ainsi : de musicien à musicien, de musicien à auditeur (et réciproquement) et d'auditeur à auditeur ${ }^{17}$.

Dans le meilleur des cas, si les conditions optimales de l'émission, de la réception et de la situation sont réunies, une sorte d' "état de grâce" est susceptible de se produire, moment privilégié dont tout musicien ou mélomane se souviendra avoir éprouvé les délices. Caractérisé par une "présence», une "inspiration", une "saveur», un «transport », un "ravissement » particulier, cet état est attesté dans de nombreuses 
cultures. Des concepts tels que le rasa en Inde, le hâl en Iran, le tarâb dans le monde arabe, le dor en Roumanie, le duende en Andalousie, la saudade ${ }^{18}$ au Portugal et au Brésil ou même le sabor cubain et le blues afro-américain sont assimilables à cet espèce de paroxysme du pouvoir de l'art, et de la musique en particulier, dont les effets se manifestent de façon tout à fait comparable sur ses émetteurs et ses récepteurs. Lorsqu'il se trouve dans cet état second, le musicien fait corps avec son instrument, et la musique le «traverse ", mue par une force indépendante de sa propre volonté. Au même titre que ses auditeurs, le musiquant devient à son tour musiqué, objet comme eux de l'« enchantement » exercé par la musique. Sans entrer dans les détails de ce processus, dont les modalités ont déjà été étudiées en profondeur, notamment dans le contexte du soufisme persan par Jean During ${ }^{19}$, bornons-nous à relever qu'il est assimilable à un ethos, voire à un élan de type mystique, suscité par le caractère magique parfois attribué à la musique.

Dans son Introduction à la sociologie de la musique, Theodor Adorno définit celle-ci comme étant «la connaissance des rapports entre l'auditeur de musique, en tant qu'individu socialisé, et la musique elle-même » (1994: 7) ; son objet premier est l'étude, « sur le plan théorique, des comportements typiques de l'écoute musicale dans les conditions de la société actuelle " (ibid.). Considérant l'écoute musicale comme un indicateur sociologique, il se pose la question de "l'adéquation ou l'inadéquation de l'écoute à ce qui est écouté ", et distingue à cet effet sept types d'auditeurs, envisagés comme autant de "profils qualitativement significatifs ", sinon toujours mutuellement exclusifs (ibid. : 10-22) :

1. L'expert, «défini selon le critère d'une écoute parfaitement adéquate. Il serait l'auditeur pleinement conscient, auquel en principe rien n'échappe, et qui, en même temps et à chaque instant, se rend à lui-même raison de ce qui est entendu»; c'est ainsi que son comportement " pourrait être qualifié d'écoute structurelle ».

2. Le bon auditeur, "qui établit spontanément des rapports, juge de façon fondée et pas uniquement d'après les catégories du prestige ou selon l'arbitraire du goût ", qui « comprend la musique un peu comme on parle sa propre langue, même s'il n'en connaît que peu ou pas du tout la grammaire et la syntaxe [...]. C'est à ce type qu'on pense lorsqu'on dit d'une personne qu'elle a le sens musical ».

3. Le consommateur de culture, qui "écoute beaucoup", qui "est bien informé», qui « collectionne les disques. Il respecte la musique comme un bien culturel, souvent comme une chose qu'il faut connaître pour son propre prestige social [...]. Son rapport avec la musique a dans l'ensemble quelque chose de fétichiste. Il consomme d'après la valeur officielle de ce qui est consommé ».

4. L'auditeur émotionnel, type qui «s'étend de celui chez qui la musique, quelle qu'elle soit, provoque des représentations et associations imagées, jusqu'à celui dont les expériences musicales se rapprochent de la rêverie indéfinie, de la somnolence; [...] pour l'auditeur émotionnel, la musique est le moyen au service des fins de sa propre économie pulsionnelle».

5. L'auditeur de ressentiment, qui «méprise la vie musicale officielle, la considérant comme creuse et vidée de sa substance», et qui, "apparemment non-conformiste dans sa protestation contre le système musical, adhère la plupart du temps, par le simple fait qu'ils existent, à des groupes et à des normes, avec toutes les conséquences socio-psychologiques et politiques ». [...] Il « se cramponne en même temps à l'idéologie du socialement supérieur, de l'élitaire, des « valeurs intérieures » ".

6. Type quantitativement le plus important, celui qui écoute la musique comme divertissement adopte de la musique une image « tout à fait du domaine de l'industrie culturelle, soit qu'elle se conforme à lui selon sa propre idéologie, soit qu'elle le crée de toutes pièces ou qu'elle le 
sollicite »[...]; « la musique pour lui n'est pas signification mais source de stimulations ». Sa relation à la musique serait de l'ordre de « l'état de dépendance ».

7. Quant à « l'indifférent à la musique, à celui qui n'a pas le sens de la musique et à celui qui est contre la musique, si l'on peut les rassembler en un seul type ", l'auteur se borne à poser l'hypothèse que le caractère réactif de tels comportements pourrait être dû à des causes de nature traumatique.

Dans la typologie ici résumée, Adorno envisage évidemment en premier lieu les auditeurs de musique classique occidentale, se référant occasionnellement aux amateurs de jazz au sein du type de l'«auditeur de ressentiment»- et à ceux des "marchandises standard» de la musique de variétés. Il ignore en revanche les auditeurs des musiques traditionnelles issues d'autres cultures que la nôtre, pour la simple et bonne raison qu'en 1962, date de la première édition allemande de son ouvrage, ceux-ci ne représentaient qu'une poignée d'originaux et que les concerts, les disques et les émissions de radio et de télévision touchant à ce domaine étaient encore des événements rarissimes.

L'élargissement de l'offre musicale ces trente dernières années à un nombre considérable de musiques jusqu'alors pratiquement inaccessibles et inconnues a été accompagné de la constitution de nouvelles catégories de publics, de goûts musicaux inédits. Sensibles aux beautés et éventuellement aux significations de telle ou telle musique "exotique », les "ethnomélomanes » constituent à cet égard un des nouveaux courants les plus significatifs de la période contemporaine. Les amateurs de musiques du monde ne représentent cependant pas une catégorie unique ; leurs orientations et leurs motivations sont probablement aussi diverses que celles dont fait état Adorno, et elles les recoupent d'ailleurs en grande partie; mais elles comportent aussi des caractères spécifiques, répondant par exemple à l'orientation géo-culturelle des goûts de chacun et aux causes psychologiques, sociales ou intellectuelles de ces goûts.

- Si l'on considère le type de l'expert, rien ne distingue en principe l'auditeur de musiques traditionnelles de celui de musiques classiques européennes, sinon l'objet de sa disposition. Il s'y caractérise plus spécifiquement par ses exigences élevées en matière d'authenticité des interprètes et des répertoires, lesquels doivent selon lui correspondre en premier lieu aux canons avérés du genre.

- Le type du bon auditeur, déjà familier de ce qu'il entend, comporte un large éventail de cas, allant du « transculturel » - qui, suite à une trajectoire personnelle, s'est immergé dans une culture étrangère, en a acquis les codes et, de ce fait, réagit plus ou moins comme s'il appartenait à cette culture - au simple mélomane ouvert, qui a développé ses affinités avant tout par l'écoute, du fait qu'il est réceptif à tout ce qui fait la particularité de la musique ou des musiques qui l'intéressent.

- Le consommateur de musique est un type assez courant parmi les amateurs de musiques traditionnelles. Son esprit collectionneur s'y manifeste par un désir de tout appréhender, par une avidité d'accroître le champ de son expérience musicale, souvent associée à une tendance systématique au comparatisme de surface. Une musique l'intéresse dans la mesure où elle confirme l'idée qu'il s'en fait.

- L'auditeur émotionnel, pour sa part, s'intéresse moins à la dimension culturelle d'une musique qu'à la stimulation sensorielle qu'elle exerce sur lui ; ce sont avant tout ses propres fantasmes qu'il y projette, même s'il croit réagir comme les autochtones ; l'effet onirique qu'une musique provoque en lui est parfois délibérément accentué par l'usage de substances psychotropes, censées le « mettre en phase » avec le moment privilégié que représente pour lui la performance musicale. 
- L'auditeur de ressentiment est aussi un type répandu dans ce domaine ; son non-conformisme s'y exprime d'autant plus librement qu'il pense y avoir trouvé le « radicalement autre ». Il est un aventurier de l'esprit, qui recherche dans ces musiques un rapport inédit au monde. L'écoute représente pour lui un voyage intérieur, et son attirance est proportionnelle à son désir de remettre en question ses habitudes culturelles et de s'ouvrir à des perspectives nouvelles. Son attirance pour telle ou telle musique se double fréquemment d'une idéalisation de ses interprètes et de leur mode de vie présumé, qui se manifeste de façon proportionnelle à son rejet des valeurs de sa propre culture.

- Celui qui écoute une musique exotique comme divertissement sera essentiellement sensible à l'ambiance qu'elle crée, et notamment aux circonstances dans lesquelles elle est présentée ou aux souvenirs qu'elle évoque pour lui - un livre, un film ou des vacances passées dans son pays d'origine, etc. La musique n'est pour lui qu'un prétexte et il n'en attend rien d'autre qu'un environnement sonore approprié à son excitabilité.

- Quant à ceux qui sont indifférents aux musiques d'ailleurs, qui n'en ont pas le sens ou qui sont contre, ils correspondent à quatre catégories distinctes. La première est de type physiologique - celui qui «n'y entend rien », quelle que soit la musique, ou qui considère ces musiques comme sans intérêt du fait de leur pauvreté ou de leur monotonie supposée. La deuxième est de type relationnel - son absence de références lui ferme l'accès à ces musiques, il n'en perçoit pas le sens, ou alors ce qu'il y perçoit ne correspond pas au message émis ${ }^{20}$. La troisième est de type psychologique - ces musiques le dérangent par leur étrangeté et il s'y refuse par peur de devoir se remettre en question. Quant à la quatrième de ces catégories, elle est de type idéologique - le rejet est avant tout un réflexe d'autodéfense à connotations éventuellement politiques, voire racistes et xénophobe.

Mais il semble bien qu'il faille ajouter à la liste proposée par Adorno deux «types », qui se manifestent fréquemment parmi les amateurs de musiques traditionnelles, et qu'on pourrait appeler respectivement l'auditeur contemplatif ou apollinien, dont le domaine de prédilection inclut volontiers les musiques orientales à prédominance modale,et l'auditeur enthousiaste ou dionysiaque, plus sensible, par exemple, aux expressions africaines et afroaméricaines caractérisées par l'usage intensif d'instruments de percussion. Ces types se retrouvent d'ailleurs à divers degrés dans d'autres courants musicaux, le premier notamment parmi les amateurs de certaines musiques classiques et anciennes, en particulier de la musique de chambre, des « concerts spirituels » et évidemment du plainchant et des musiques religieuses, le second surtout parmi ceux de jazz, de rock et de leurs dérivés.

61 Pour ces deux types d'auditeur - le contemplatif et l'enthousiaste - le concert ou l'événement musical est vécu comme une sorte de rite. Dépassant le niveau de la simple jouissance sensorielle ou intellectuelle, leur écoute est de nature essentiellement participative ; consciemment ou non, elle procède d'une sorte de quête de sacralité. Pour le premier, cette quête se manifeste par un désir d'extase et d'identification mystique au soi le plus profond; pour le second, à l'autre pôle, par une volonté de libération des contraintes de l'ego par des moyens de l'ordre de ceux mis en œuvre dans les phénomènes de transe. On se rappellera à cet égard la distinction relevée entre ces deux termes par Gilbert Rouget, qui réserve "extase " «à un certain type d'états, disons seconds, atteints dans le silence, l'immobilité et la solitude, » et qui désigne par «transe » «ceux qui ne s'obtiennent que dans le bruit, l'agitation et la société des autres » (1990: $47)^{21}$. 
L'appolinien recherche donc plutôt l'extase dans la mesure où, en tant que «méthode douce » œuvrant dans une logique de continuité par rapport aux états de conscience ordinaires, elle correspond à un cheminement qu'on pourrait qualifier de "mélodique » ou de « linéaire »; le dionysiaque, quant à lui, est plus sensible à la « méthode forte » que représente la transe en tant que processus caractérisé par sa discontinuité et sa nature "rythmique», faite de ruptures, mais aussi organisée de façon cyclique ${ }^{22}$. Il convient toutefois de relever que ces deux types ne manifestent que des tendances psychologiques; quelle que soit la sincérité des individus qui y répondent, elles demeureront à l'état latent ou ne se manifesteront que de façon fugitive si elles ne sont pas assorties de l'acquisition de méthodes cognitives appropriées du type de celles, d'ordre souvent initiatique, qu'on rencontre au sein de nombreuses traditions spirituelles 23 .

\section{Nous et les autres ${ }^{24}:$ un jeu de miroirs}

62 De manière générale, le concert de musique traditionnelle est pour l'auditeur une occasion de rencontrer une culture à travers une expression dont il attend volontiers, nous l'avons vu, une image de pureté et d'authenticité; son attrait pour ce domaine procède souvent d'un désir de retour aux sources, s'inscrivant parfois dans une démarche plus globale de remise en question de son propre système de valeurs. L'amateur se rend rarement à une manifestation de ce genre en consommateur passif, mais plutôt avec un désir de participation et d'identification à ce qui lui est proposé procédant d'une démarche esthétique aux connotations parfois politiques ou spirituelles. Il perçoit dans les musiques «d'ailleurs" vers lesquelles le guident ses affinités les échos d'une aspiration de son être intime; ces musiques manifestent pour lui un idéal auquel il n'a ordinairement pas accès. Il y cherche une confirmation de ses propres intuitions.

Quant aux musiciens qui se prêtent au jeu du concert, celui-ci constitue pour eux une forme utile, parfois nécessaire, de promotion et de valorisation de leur art. Dans de nombreux cas, les structures de patronage dont ils bénéficiaient au sein de leur contexte traditionnel ont disparu ou se sont modifiées à tel point qu'elles ne leur permettent plus de maintenir leur pratique comme par le passé. L'intérêt manifesté par l'étranger leur apparait donc non seulement comme une stimulation bienvenue, mais aussi comme une source appréciable de revenus. Certains genres, nous l'avons évoqué, doivent même leur survie au surgissement de ces nouveaux débouchés. La situation est évidemment paradoxale, mais elle exprime à sa manière l'état de dépendance économique du reste du monde vis-à-vis des pays qui ont encore les moyens de s'offrir le luxe d'une culture subventionnée. Cette perspective peut-elle seule offrir les conditions d'un renouveau pour des traditions musicales dont certaines sont actuellement fragilisées par la rapidité et la brutalité des mutations qui les affectent, ou est-elle tout au plus une sorte de bouée de sauvetage, aussi providentielle que provisoire?

64 "Il est assez facile de parler du renouveau de la musique traditionnelle », relevait déjà en 1964 l'ethnomusicologue Peter Crossley-Holland. "Mais sans deux conditions esssentielles, tout cela n'est qu'un rêve vide. Ces conditions sont d'abord le renouveau de la société traditionnelle elle-même et, ensuite, la manifestation du génie. Admettons franchement que nous ne disposons d'aucun de ces deux éléments » (1964: 18). La situation est aujourd'hui sensiblement différente dans la mesure où l'élargissement de l'intérêt pour ce domaine est devenue porteur d'enjeux nouveaux, y compris sur les plans 
psychologique, politique, social et économique. L'interaction des musiques traditionnelles du monde et de leurs nouveaux publics se développe comme un jeu de miroirs dans lequel chacun cherche dans l'autre le reflet de son propre idéal: besoin de prestige et de ressources d'une part, quête d'authenticité et d'ouverture de l'autre. Cette relation témoigne d'une redéfinition des rôles, dans laquelle ce ne sont plus nous, mais les autres qui ont valeur d'exemplarité.

Mais il serait faux de croire qu'un musicien traditionnel n'est que le porte-parole des valeurs de sa culture. Le talent individuel de grands interprètes passés et présents est évidemment pour beaucoup dans le développement des critères d'excellence d'une musique ; lorsque nous croyons écouter la manifestation d'un génie ethnique immuable et intemporel, ce que nous apprécions est en fait souvent la démarche personnelle - et unique en tant que telle - d'un artiste inspiré ou novateur dont, faute des critères nécessaires, nous ne percevons pas toujours l'originalité. Que son jeu respecte ou non les règles de la tradition qui le sous-tend est une chose, mais la présence ou l'absence de talent demeure en toute circonstance la condition première de l'efficacité de la musique. Quels que soient sa culture, l'idiome dans lequel il s'exprime et le lieu où il se produit, le musicien demeure un artiste dans le plein sens du terme et, selon le degré de sa créativité et de son inspiration, il est capable ou non d'émouvoir et de convaincre ceux à qui il s'adresse.

\section{BIBLIOGRAPHIE}

ADORNO Theodor W., 1994 [1962], Introduction à la sociologie de la musique. Douze conférences théoriques. Traduit de l'allemand par Vincent Barras et Carlo Russi. Genève : Contrechamps Editions.

AUBERT Laurent, 1991a, « La voix des ancêtres. La musique comme fait humain ». In Laurent Aubert éd. : Mondes en musique. Genève : Musée d'ethnographie : 11-21.

AUBERT Laurent, 1991b, Musiques traditionnelles. Guide du disque. Genève : Georg.

AUBERT Laurent, 1991c, « La World Music, dernière tentation de l'Occident ». Le Monde, 17 octobre 1991., 1995, « Les ailleurs de la musique. Paradoxes d'une société multiculturelle». Internationale de l'imaginaire, nouvelle série, $4:$ « La musique et le monde » : 13-25.

BLACKING John, 1980 [1973], Le sens musical. Traduit de l'anglais par Eric et Marika Blondel. Paris : Les Editions de Minuit.

BRAILOIU Constantin, 1960, «La vie antérieure ». In Roland Manuel éd. : Histoire de la musique 1.

Encyclopédie de la Pléiade. Paris : Gallimard : 118-127.

BURCKHARDT Titus, 1969, Introduction aux doctrines ésotériques de l'Islam. Paris : Dervy-Livres.

COOMARASWAMY Ananda K., 1990 [1943], La philosophie chrétienne et orientale de l'art. Traduit de l'anglais par Frédéric Monneyron. Puiseaux: Pardès.

CROSSLEY-HOLLAND Peter, 1964, «Preservation and renewal of traditional music ». Journal of the International Folk Music Council, XVI: 15-18. 
DURING Jean, 1990, Musique et extase. L'audition mystique dans la tradition soufie. Paris : Albin Michel.

DURING Jean, 1994, Quelque chose se passe. Le sens de la tradition dans l'Orient musical. Lagrasse (France) : Verdier.

ECO Umberto, 1984 [1968], La structure absente. Introduction à la recherche sémiotique. Traduit de l'italien par Uccio Esposito-Torrigiani. Paris : Mercure de France.

KHAZNADAR Chérif et Michel de LANNOY, 1995, « Les trois voies de la musique. Entretien avec Chérif Khaznadar » par Michel de Lannoy. Internationale de l'imaginaire, nouvelle série, $4:$ : La musique et le monde $»$ :37-57.

LORTAT-JACOB Bernard, 1994, Musiques en fête. Maroc, Sardaigne, Roumanie. Collection Hommes et musiques 1. Nanterre : Société d'ethnologie.

LECOMTE Henri, 1992, « L'avant-dernière tentation de l'Occident ». Trad Magazine, 20 : 28.

NATTIEZ Jean-Jaccques, 1987, Musicologie générale et sémiologie. Paris : Christian Bourgeois.

PEARSON Mike, 1996, « Réflexions sur l'ethnoscénologie », traduit de l'anglais par Pierre Bois. Internationale de l'imaginaire, nouvelle série, $5:$ « La scène et la terre » : 55-64.

ROUGET Gilbert, 1990 [1980], La musique et la transe. Esquisse d'une théorie générale des relations de la musique et de la possession. Nouvelle édition revue et augmentée. Préface de Michel Leiris. Paris : Gallimard.

SAKATA Hiromi Lorraine, 1994, « The Sacred and the Profane: Qawwâlî Represented in the Performances of Nusrat Fateh Ali Khan ». The World of Music, 36/3 : 86-99.

SCHAEFFER Pierre, 1966, Traité des objets musicaux. Essai interdisciplinaire. Nouvelle édition. Paris : Seuil.

TODOROV Tzvetan, 1989, Nous et les autres. La réflexion française sur la diversité humaine. Paris : Seuil, collection Points-Essais 250.

\section{NOTES}

1. Comme la musique classique européenne et celles de plusieurs civilisations asiatiques, l'expression des griots du Mali peut effectivement prétendre à ce statut, par opposition à celui de musique "populaire ", dans la mesure où elle est directement issue de l'art de musiciens de cour, attachés au service de la noblesse de l'ancien empire Mandingue.

2. Dans sa traduction des «Réflexions sur l'ethnoscénologie » de Mike Pearson, Pierre Bois écrit en note: "Nous conservons à dessein le terme anglo-saxon de performance dont le champ sémantique investit aussi bien le rite, le jeu, le théâtre, la musique, et n'a pas d'équivalent français » (Pearson $1996: 55$, n. 1). Les termes d'« exécution » ou d'« interprétation » excluent la musique (ainsi que la danse ou le théâtre) en tant que devenir ou que processus, ils impliquent nécessairement que le musicien, reproduit à sa façon une composition préétablie. Or à l'évidence, cette séparation entre l'acte de la composition et celui du jeu musical n'est pas universellement pertinente. Quant à " prestation », son sens est limité à ce qu'un artiste (ou un athlète) produit pour un public. Plutôt que de continuer à «tourner autour du pot», je propose d'adopter «performance » une fois pour toutes en français. Ceci se justifie d'autant plus que, selon le Petit Robert, le mot anglais vient de l'ancien français parformance qui, au $\mathrm{XVI}^{\mathrm{e}}$ siècle, signifiait « accomplissement » ou « exécution ». 
3. Dans un article précédemment publié, j'avais relevé six caractéristiques partagées par les musiques traditionnelles selon Constantin Brailoiu. Il les considérait comme : 1. intelligibles, 2. au delà des caprices du goût, 3. liées à des croyances, 4. fonctionnelles, 5. puissantes et 6 . pérennes ( $c f$. Brailoiu $1960: 122$ et Aubert 1991a : 14-19).

4. Ce problème des limites de la tradition a déjà été abordé dans une précédente publication (Aubert 1991b : XII-XIV).

5. La formule est de John Blacking (1980:18).

6. Pour une définition du terme $d^{\prime}$ ' authenticité » dans ce domaine, voir notamment l'analyse très pertinente qu'en fournit Jean During (1994 : 189-209).

7. Parus notamment dans la prestigieuse collection Ocora/Radio France, dans celle de Peter Gabriel, Realworld, chez Victor-Japon ou encore dans la série des Oriental Star Agencies Ltd., ces disques figurent effectivement parmi les plus grosses ventes de tous les temps dans le domaine des musiques traditionnelles ( $c f$. la discographie de l'article de Pierre-Alain Baud ici même).

8. Il est intéressant de noter que Nusrat Fateh Ali Khan fut le premier à introduire la pratique virtuose de la solmisation (sargam) dans son interprétation du qawwâlî, un usage traditionnellement réservé aux genres de musique savante profane tels que le khyâl; cette innovation est d'ailleurs réprouvée au Pakistan par certains milieux orthodoxes soufis en raison de son caractère spectaculaire et gratuit, jugé incompatible avec les principes du samâc (audition spirituelle) qui sont l'essence même du qawwâlî.

9. Mais le pire est peut-être encore à venir, comme le laisse supposer un article paru le 26 mai 1996 dans le quotidien Le Matin sous le titre «Mirages d'Orient ». Témoin ironique d'une dérive programmée, le journaliste Vincent Borcard y constate, à propos du dernier CD de Nusrat, Night Song (Real World), que « le résultat est sympathique, même si la musique fait penser par instants à Jean-Michel Jarre. A l'avenir, des duos avec Frank Sinatra, Pavarotti ou Dalida sont àcraindre »...

10. Cf. infra, "L'art de bien entendre ", pour l'opposition entre une écoute apollinienne et une écoute dionysiaque.

11. De nombreux exemples montrent qu'une tournée à l'étranger peut avoir des effets positifs sur l'avenir d'une troupe ou d'un genre artistique, voire d'une communauté ou d'une minorité ethnique entière. Elle représente une forme de promotion à laquelle les instances culturelles du pays concerné sont souvent sensibilisées du fait des enjeux politiques qu'elle peut comporter. Dans d'autres cas, ce n'est que grâce à la stimulation provoquée par l'intérêt de responsables culturels internationaux qu'une tradition artistique a pu échapper à la disparition. Voir à ce propos Khaznadar et de Lannoy (1995).

12. Voir aussi son article ici même.

13. L'inverse est aussi fréquent, et des musiciens se livrant dans leur propre contexte à toutes sortes d'innovations, soit par goût personnel, soit pour des raisons économiques, se posent en champions de la tradition dès lors qu'ils se produisent en Occident, afin de répondre aux attentes du public qu'ils visent.

14. Ces expressions sont empruntées à Gilbert Rouget.

15. On constate depuis quelques années que ce courant de recherche et d'interprétation des formes anciennes est en train de s'étendre à d'autres cultures telles que celles de la Turquie et du monde arabe classiques, de l'Asie orientale (Chine, Corée, Japon et Vietnam) et même de l'Antiquité grecque ou égyptienne et des civilisations pré-hispaniques d'Amérique du Sud.

16. $C f$. note 5 .

17. Pour une approche appliquée au domaine de l'anthropologie du théâtre et de l'ethnoscénologie, voir notamment Pearson (1996).

18. Il est intéressant de noter que saudade vient de l'arabe sawda, qui signifie "nostalgie", « mélancolie ». 
19. A propos du hâl dans le contexte persan, qu'il définit comme étant une «"modification momentanée de l'état de conscience ", "sortie de l'état normal ", mais dans le sens d'une valorisation » (1994: 162), cet auteur relève que « les enjeux les plus importants du hâl semblent être d'une part la fusion, l'unification qu'il établit entre le sujet musiquant et la musique, et d'autre part sa propriété d'ouvrir les chemins de la création » (ibid. : 165).

20. Dans un texte sur la rhétorique, Umberto Eco parle à ce propos d'un «décodage "aberrant»", qui fait que le destinataire "reçoit un message dans une situation psychosociologique qui n'est pas prévue par l'émetteur. Celui-ci s'est fondé sur des codes, des souscodes et des connaissances supposées chez le destinataire, mais que le destinataire ne possède pas ; celui-ci se reporte au contraire à des codes privés, à des champs sémantiques d'un autre type, ou il subit l'impact de connotations aléatoires, victime souvent de circonstances déviantes » (1984: 166).

21. L'extase, écrit Rouget, " est une expérience dont on garde, souvent de manière aiguë, le souvenir, sur laquelle on peut revenir à loisir après coup et qui ne donne pas lieu à cette dissociation qui caractérise la transe ». La transe, au contraire, "a pour caractéristique d'être sujette à une amnésie totale. Le rapport du moi à sa transe est donc, à cet égard, totalement inverse de celui du moi à son extase " (1990: 49). "La transe apparaît donc toujours, d'une manière ou d'une autre, comme un dépassement de soi-même, comme une libération résultant de l'intensification d'une disposition mentale ou physique, bref comme une exaltation automutilatrice parfois - du moi » (ibid. : 57-58).

22. Jorge López Palacio me faisait remarquer à ce propos que ces deux types pouvaient aussi être définis respectivement comme "l'assoiffé » et "l'affamé » de musique, la soif de musique induisant à l'extase et la faim de musique à la transe. Il proposait en outre la distinction entre l'état «liquide» de la mélodie - ou d'une musique à prépondérance mélodique - et l'état «solide» du rythme - ou d'une musique où celui-ci est prédominant (communication personnelle).

23. Le soufisme distingue à cet égard le hâl ou "état " spirituel, du maqâm ou "station "; le premier, considéré comme passager, étant accessible à tout un chacun, et le second, considéré comme stable, en principe réservé aux seuls initiés (cf. Burckhardt 1969 : 119-120, et supra, n. 16).

24. Titre de l'ouvrage de Todorov cité en référence (1989).

\section{RÉSUMÉS}

Cet article aborde la question de la place des musiques dites traditionnelles dans notre société. Dans le premier chapitre, l'auteur s'interroge sur les critères de la tradition en musique, et notamment sur le problème de limites que pose ce concept dès lors qu'on tend à l'appliquer globalement. Les musiques traditionnelles constituent-elles réellement une catégorie en soi, ou ne s'agit-il que d'un «fourre-tout " commode, mais impropre à définir l'ensemble de ses composantes supposées ? L'accent est ensuite mis sur le phénomène du concert en tant que mode de représentation de musiques qui n'y sont a priori pas destinées. Ces musiques conservent-elles leur authenticité et leur raison d'être en l'absence de leur système habituel de références, ou leur représentation sur scène n'est-elle qu'une évocation, voire une trahison par le fait qu'elle privilégie certains aspects de la performance et qu'elle en exclut d'autres? Le concert implique certaines adaptations, il modifie le rapport entre «musiquants» et "musiqués ", créant volontiers une disparité entre les motivations de l'émetteur de musique et celles du récepteur. 
L'observation des comportements des publics conduit ensuite l'auteur à brosser l'esquisse d'une typologie générale de l'auditeur, et enfin à constater qu'un nouveau type de relation entre nous et les autres est en train de s'instaurer, dans lequel la musique semble jouer un rôle essentiel.

This article tackles the question of the role traditional music plays in our society. In the first chapter the author looks at criteria for assessing tradition in music and more specifically the limits placed on them when they are applied worldwide. Does 'traditional music' really constitute a separate category, or is the term simply a convenient 'hold-all' inadequate when it comes to defining its different parts? The emphasis in this article is then placed on the concert as a mode of performance for traditional music, a setting for which it was not designed. In the absence of their usual systems of reference, do different types of traditional music preserve their authenticity or is not an on-stage performance a mere hint of what was, or even a treacherous rendering of what was, given that some aspects are pushed to the fore and others excluded? Implicit in the term concert is adaptation, and adaptation changes the rapport between those playing and those played to, thus gladly creating a motivational disparity between broadcasters and receivers. An observation of audience behaviour then leads the author to proceed with a rough outline typology of the concert-goer and finally to state that a new relationship between 'us' and 'the others' in which music plays an important role is being forged.

\section{AUTEUR}

\section{LAURENT AUBERT}

Laurent Aubert a une formation d'ethnomusicologue et de musicien. Il est le fondateur et l'actuel directeur des Ateliers d'ethnomusicologie ; il est également conservateur au Musée d'ethnographie de Genève, où il s'occupe des collections d'instruments de musique et des Archives internationales de musique populaire (AIMP). Après des recherches de terrain, notamment en Inde et au Népal, il a travaillé à une réévaluation de l'œuvre de Constantin Brâiloiu. Ses préoccupations actuelles concernent essentiellement la problématique des musiques en migration, à laquelle il contribue par l'organisation de manifestations (concerts, festivals, colloques, stages...) et par une action de soutien aux musiciens résidant en région genevoise. Il est l'auteur de plusieurs publications (livres, articles, CD) touchant à ces différents domaines et collabore régulièrement au mensuel Le Monde de la Musique. 